\title{
Hiperbilirrubinemia neonatal
}

\author{
Dres. Carmen Díaz Quiroz*, Jorge Gutiérrez Pueima*
}

La etapa neonatal es el período de la vida en que la presencia de ictericia es más frecuente, debido a factores propios del recién nacido (poliglobulia, hemólisis fisiológica, inmadurez hepática, etc.). Así, durante la primera semana de vida, aproximadamente el $80 \%$ de los recién nacidos pretérmino y el $50 \%$ de los recién nacidos de término normales la presentan.

En determinadas circunstancias (incompatibilidad $\mathrm{RH}$, incompatibilidad $\mathrm{ABO}$, etc.) esta ictericia traspone las barreras de lo fisiológico para convertirse en una patología de riesgo importante, en cuanto al pronóstico vital inmediato y a las repercusiones neurológicas a futuro de los pacientes afectados.

La bilirrubina indirecta libre (no unida a albúmina) es la responsable de la neurotoxicidad.

Se ha destacado repetidamente que no hay concentración crítica de bilirrubinemia que pueda utilizarse como criterio arbitrario en cuanto a producir secuelas neurológicas. Así, mientras en el recién nacido de término sano es excepcional ver daño neurológico con valores de bilirrubinemia inferiores a $20 \mathrm{mg} \%$, la aparición de lesión cerebral relacionada con la bilirrubina se ha comprobado repetidamente en recién nacidos pretérminos de bajo peso al nacer con concentraciones séricas de bilirrubina $\tan$ bajas como 6 a $9 \mathrm{mg} \%$. En ellos influyen, además de la prematuridad, factores que frecuentemente se presentan en el pretérmino y que conocidamente aumentan el riesgo de Kernicterus, como son hipoxia,

*Servicio Pediatría, Hospital Regional Dr. Leon ardo Guzmán. acidosis, hipotermia, hipoalbuminemia, hipoglicemia e infección.

Ante la importancia del problema, se ha utilizado, a través del tiempo, distintos métodos terapéuticos: exanguineotransfusión, que sigue siendo el método más efectivo en disminuir rápidamente los niveles de bilirrubinemia, pero que cuenta con riesgos propios del procedimiento que incluso presenta una mortalidad del 1 al $6 \%$ según los distintos autores; fototerapia, que en los últimos años ha demostrado ser eficaz, y presentar complicaciones mínimas a corto plazo, y aún no totalmente aclaradas a largo plazo (efectos sobre crecimiento y desarrollo); fenobarbital, de menor utilidad debido a la latencia propia de todo mecanismo de inducción enzimática y posible toxicidad a largo plazo.

Actualmente, si bien se ha avanzado en el manejo de la hiperbilirrubinemia, persiste el problema de determinar en forma segura cuál es el riesgo de encefalopatía bilirrubínica en cada caso individual. Lo ideal sería poder contar, en el futuro, con un examen de laboratorio sencillo que permitiera medir la bilirrubina libre, o indirectamente la capacidad de saturación de la albúmina plasmática, a fin de tener la certeza, en cada caso clínico, cuál es su riesgo de encefalopatía $y$ poder tratarlo oportun amente.

Hay en el momento actual varios métodos que intentan medir este riesgo en forma segura, pero ninguno de ellos tiene aún el rendimiento suficiente para aplicarlo rutinariamente en la práctica clínica y confiarle el futuro neurológico e incluso vital de nuestros 
pacientes. Considerando la frecuencia e importancia de la hiperbilirrubinemia durante el período neonatal, se decidió determinar la magnitud de esta patologia en nuestro medio, su etiología, tipo de pacientes en que se presenta y curva de bilirrubina de ellos además de valorar la utilidad y riesgo de los métodos empleados en su tratamiento: fototerapia $y$ exanguin eotransfusión.

\section{MATERIAL Y METODO}

Se revisaron 180 fichas clínicas de casos de hiperbilirrubinemia correspondientes a pacientes hospitalizados en la Unidad de Recién Nacidos Patológicos y Prematuros del Hospital Regional Dr. Leonardo Guzmán de Antofagasta durante el quinquenio 1973-1977. Se definió el criterio de selección de hiperbilirrubinemia como una bilirrubinemia superior a $12 \mathrm{mg} \%$ durante la primera semana de vida. Se analizó las bases fisiopatológicas de los métodos terapéuticos empleados aclarando sus normas de aplicación en el material estudiado.

\section{RESULTADOS}

Primeramente presentamos en la Tabla 1 la magnitud del problema en nuestro Servicio. Así vemos que de 3.500 a 4.100 RN vivos/año ingresan a la Unidad de RN patológicos y prematuros entre el 12,9 y $15,6 \%$; de estos ingresos, el 6 a $10 \%$ corresponde a casos de hiperbilirrubinemia, definiendo como tal toda cifra de bilirrubinemia total supe- rior a $12 \mathrm{mg} \%$ durante la primera semana de vida, cualquiera sea su etiología. Cabe hacer notar que si bien la morbilidad por hiperbilirrubinemia, su mortalidad es casi nula, ya que de los 180 casos revisados sólo se consigna un fallecimiento $(0,55 \%)$, el que corresponde a un RNPT de menos de 36 semanas de edad gestacional con enfermedad hemolítica por $\mathrm{Rh}$ grave que presenta asociado un SIDRI y que fallece al cuarto día de vida después de haber sido sometido a una exanguineotransfusión al segundo día de nacido.

Tabla 1

NUMERO DE RN VIVOS, INGRESOS A LA UNIDAD RN PATOLOGICO Y PREMATUROS Y MORBILIDAD POR HIPERBILIRRUBINEMIA

\begin{tabular}{lccc}
\hline Año & $\begin{array}{c}\text { RN Vivos } \\
N^{\circ} \text { casos }\end{array}$ & $\begin{array}{c}\text { Morbilidad } \\
N^{\circ} \text { casos }\end{array}$ & $\begin{array}{c}\text { Mor hiperbilir. } \\
N^{\circ} \text { casos }\end{array}$ \\
\hline 1973 & 4.104 & 534 & 37 \\
1974 & 4.004 & 563 & 33 \\
1975 & 3.875 & 607 & 34 \\
1976 & 3.781 & 508 & 30 \\
1977 & 3.513 & 455 & 46 \\
\hline Total & 19.277 & 2.667 & 180 \\
\hline
\end{tabular}

En la Tabla 2 y Gráfico 1 vemos la etiología de los casos de hiperbilirrubinemia catalogada en cuatro grupos: Enfermedad hemolítica por $\mathrm{Rh}$, enfermedad hemolítica por $\mathrm{ABO}$, prematuridad y otros; este último grupo consigna predominantemente casos de reabsorción de colecciones hemáticas además de casos secundarios a poliglobulia y causas no precisadas.

Tabla 2

EIIOLOGIA DE HIPERBILIRRUBINEMIA

\begin{tabular}{|c|c|c|c|c|c|c|c|c|c|c|}
\hline \multirow{3}{*}{ Año } & Enf. & $\mathrm{Hem}$ & Enf. & Hem. & \multicolumn{2}{|c|}{ Premat. } & \multicolumn{2}{|c|}{ Otros } & \multicolumn{2}{|c|}{ Total } \\
\hline & \multicolumn{2}{|c|}{$x R h$} & \multicolumn{2}{|c|}{$x A B O$} & & & & & & \\
\hline & $N^{\infty}$ & $\%$ & $N^{\circ}$ & $\%$ & $N^{\infty}$ & $\%$ & $N^{\circ}$ & $\%$ & $N^{\circ}$ & $\%$ \\
\hline 1973 & 8 & 21,6 & 5 & 13,5 & 18 & 48,7 & 6 & 16,2 & 37 & 100 \\
\hline 1974 & 5 & 15,2 & 6 & 18,2 & 18 & 54,6 & 4 & 12,0 & 33 & 100 \\
\hline 1975 & 14 & 41,2 & 7 & 20,6 & 5 & 14,7 & 8 & 23,5 & 34 & 100 \\
\hline 1976 & 7 & 23.3 & 11 & 36,7 & 8 & 26,7 & 4 & 13,3 & 30 & 100 \\
\hline 1977 & 7 & 15,2 & 19 & 41,3 & 13 & 28,3 & 7 & 15,2 & 46 & 100 \\
\hline Total & 41 & 22,8 & 48 & 26,7 & 62 & 34,4 & 29 & 16,1 & 180 & 100 \\
\hline
\end{tabular}


Gráfico 1

\section{ETIOLOGIA DE HIPERBILIR RUBINEMIA}

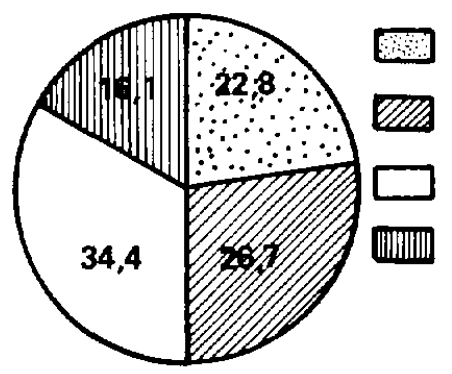

Enf. Hemolítica x Rh

Enf. Hemolítica x ABO

Prematuridad

El $22,8 \%$ de los casos corresponde a enfermedad hemolítica por $\mathrm{Rh}$; esta etiología muestra un ascenso de su frecuencia en 1973 a 1975 para disminuir posteriormente, tal vez en relación a un mejor control obstétrico.

El $26,7 \%$ de los casos corresponde a enfermedad hemolítica por ABO; esta etiologia presenta un aumento creciente de 1973 a 1977. De ellos, el 80 a $86 \%$ corresponde a incompatibilidad O-A y sólo el 14 a $20 \%$ a incompatibilidad O-B.

El $34,4 \%$ de los casos corresponde a hiperbilirrubinemia del pretérmino; esta etiolo- gía presenta su máximo en los primeros dos años revisados para estabilizarse posteriormente en 27 a $28 \%$.

El $16,1 \%$ de los casos corresponde al rubro "otros"; esta etiología presenta un pick en 1975, mostrando en los otros años revisados una frecuencia más o menos estable de 12 a $16 \%$.

El Gráfico 2 muestra la relación entre edad gestacional y etiología de la hiperbilirrubinemia.

Se aprecia que tanto las enfermedades hemolíticas por $\mathrm{Rh}$ como por $\mathrm{ABO}$ acrecientan al aumentar la edad gestacional y por lo tanto el tiempo de sensibilización; la hiperbilirrubinemia por prematuridad se presenta en nuestro material con igual frecuencia en los menores de 36 semanas y en los de 36 a 38 semanas de edad gestacional; el rubro otros, por considerar especialmente patología traumática, es más frecuente en los mayores de 38 semanas, que al presentar un mayor peso de nacimiento tienen más posibilidad de partos traumáticos.

Otra variable que nos interesó analizar fue la curva de hiperbilirrubinemia de las distintas etiologías; esto sólo fue posible en lo que respecta al momento de aparición

Gráfico 2

\section{RELACION ENTRE EDAD GESTACIONAL Y ETIOLOGIA DE HIPERBILIRRUBINEMIA}

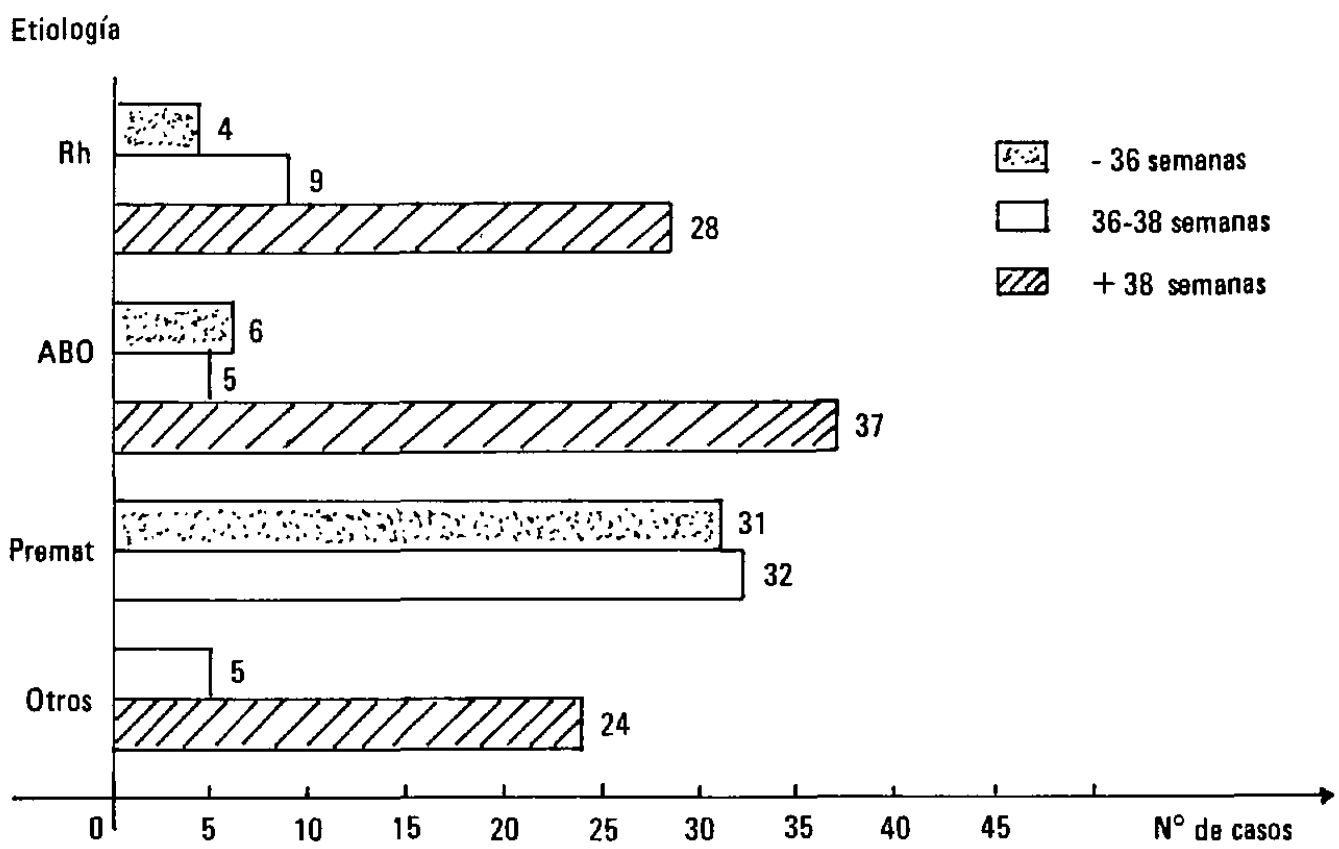


de la ictericia, ya que tanto el momento y magnitud de su máximo, como el del inicio de su descenso fue muy variable, viéndose influido por el tratamiento empleado. Así, determinamos que la enfermedad hemolítica por Rh presentó ictericia precoz (aparecida antes de las 24 horas de vida) en el $71-86 \%$ de los casos; la enfermedad hemolítica por $\mathrm{ABO}$ sólo en el 20-60\% de los casos, y no hubo ocurrencia de ictericia precoz en hiperbilirrubinemia del pretérmino ni otros.

Antes de pasar a revisar los resultados obtenidos con respecto al tratamiento de la hiperbilirrubinemia neonatal, haremos un somero análisis de las bases fisiopatológicas de los métodos terapéuticos empleados en nuestro Servicio y sus normas de aplicación en el material estudiado.

A. Exanguineotransfusión o recambio sanguíneo: fue el primer método utilizado, y sigue siendo el más efectivo para lograr un rápido descenso de los niveles séricos de bilirrubina. Se basa en remover la bilirrubina que está circulando unida a albúmina y parte de la que está en los tejidos, logrando su redistribución en el espacio vascular.

Sus indicaciones, en relación a hiperbilirrubinemia, en nuestro Servicio son:

a) Valores de bilirrubina de cordón sobre $4 \mathrm{mg} \%$.

b) Aumento del nivel de bilirrubina sérica a un ritmo superior a $0,5 \mathrm{mg} /$ hora, durante el primer día de vida.

c) Bilirrubinemia mayor a $20 \mathrm{mg} \%$ en el RNT sin patología asociada, a partir del $2^{\circ}$ día de vida.

d) Bilirrubinemia mayor a $18 \mathrm{mg} \%$ en el RNPT $\sin$ patología asociada, a partir del $2^{\circ}$ día de vida.

e) Bilirrubin emia $2 \mathrm{mg} \%$ bajo los niveles mencionados anteriormente (c y d) en caso de factores asociados que aumenten el riesgo de Kernicterus, ej: acidosis, hipoxia, hipoglicemia, hipoalbuminemia, hipotermia, infección.

f) Con cualquier cifra de bilirrubinemia ante la presencia clínica de Kernicterus.

Como normas para la realización de la exanguín eotransfusión consideramos:

1. Uso de sangre temperada a $37^{\circ} \mathrm{C}$.

El tipo de sangre a usar depende de la causa que in dicó la exanguíneotransfusión.
- En la incompatibilidad $\mathrm{Rh}$ se usa sangre $\mathrm{Rh}(-)$, del mismo grupo clásico del $\mathrm{RN}$ o, en su defecto, O IV Rh (-).

- En la incompatibilidad ABO se usa sangre O IV del mismo Rh del RN.

- En la hiperbilirrubinemia del RNPT se usa sangre del grupo y $\mathrm{Rh}$ del $\mathrm{RN}$.

2. Volumen sanguíneo de $180-200 \mathrm{cc} / \mathrm{kg}$ (2 volemias).

3. Uso de sangre fresca, de no más de 48 horas de extraída, porque luego de ese plazo disminuye significativamente la vida media de los glóbulos rojos, baja el $\mathrm{pH}$ sanguíneo y aument a la kal emia.

4. Uso de sangre heparinizada, citratada, calcificada. A la sangre de Banco se adiciona $1.500 \mathrm{U}$ de heparina por cada $500 \mathrm{cc}$ de sangre $y$ : cc de gluconato de calcio al $10 \%$ por cada 100 cc de sangre recambiada.

5. Paciente en ayunas 3-4 horas antes del procedimiento o realización de lavado gástrico.

6. Realización del procedimiento a través de vena umbilical, según técnica quirúrgica.

7. Toma de muestras de bilirrubinemia pre y postrecambio.

8. Evitar hipotermia del RN durante el procedimiento. Controlar su aspecto y ciclo vital periódicamente y tener a mano equipos de resucitación, administración . de $\mathrm{O}_{2}$ e intubación para enfrentar cualquier pos̀ible complicación.

9. Uso de antibióticos sólo en caso de infección manifiesta, en caso de procedimiento técnicamente laborioso que los justifique $o$ a partir del $2^{\circ}$ recambio en adelante.

10. Uso de fototerapia postrecambio para aminorar la cuantia del rebote y disminuir la posibilidad de efectuar un nuevo recambio.

B. Fototerapia o luminoterapia: su uso ha aumentado y ha recibido gran atención en los últimos años. Ha demostrado su utilidad, tanto en el uso profiláctico como terapéutico, de la hiperbilirrubin emia.

Su mecanismo de acción no es totalmente conocido. Se basa en la fotodescomposición, a nivel de la piel, de la bilirrubina indirecta en un pigmento semejante a la biliverdina que por su condición de hidrosoluble es excretado por vía renal, no requiriéndose conjugación a nivel hepático. 
En nuestro Servicio utilizamos el procedimiento tanto en la hiperbilirrubinemia del RNPT como en las incompatibilidades ABO y Rh. Para su administración utilizamos un aparato que consta de 10 tubos con lámparas de 20 Watt de luz blanca, ubicado a $80 \mathrm{~cm}$. del paciente.

Como normas durante el procedimiento observamos:

1. Paciente desnudo en cuna o incubadora.

2. Paciente con ojos cubiertos durante el procedimiento para proteger la retina; descubrirlos durante la alimentación para producir estimulación neuroendocrina.

3. Control periódico de temperatura del paciente para evitar enfriamientos o sobrecal entamientos.

4. Cambios frecuentes de posición para variar la superficie cut ánea expuesta a la luz.
5. Control periódico de bilirrubinemia, ya que la fototerapia elimina la ictericia cutánea impidiendo la evaluación clínica de la hiperbilirrubinemia.

6. Esquema de tratamiento variable en indicación (preventiva o terapéutica) y duración alterna cada 12 horas o continua por 3-6 días, basándose en estudios que no muestran diferencias significativas en la eficacia de los distintos esquemas utilizados.

7. Suspensión de tratamiento ante curva de bilirrubinemia en descenso o valores de bilirrubina sérica inferiores a $12 \mathrm{mg} \%$.

8. Control de bilirrubinemia a las 12 horas de suspendida la fototerapia para evaluar eventual rebote y ratificar o rectificar la suspensión de ésta.

El Gráfico 3 nos muestra el uso de exanguineotransfusión y fototerapia en los casos de hiperbilirrubinemia revisados.

Gráfico 3

\section{METODOS TERAPEUTICOS EN HIPERBILIRRUBINEMIA}

\section{Etiología}

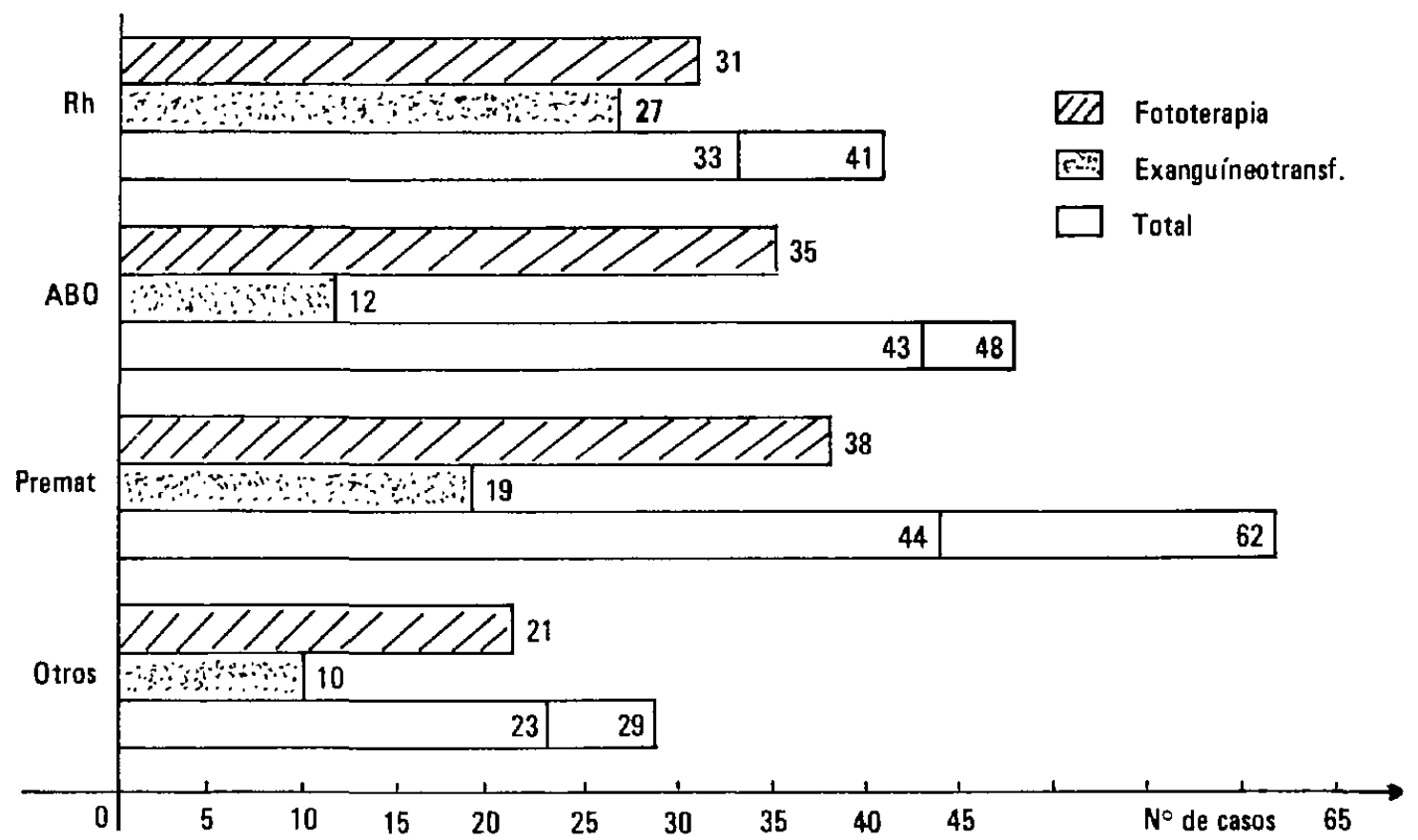

Nota: durante 1973 no se empleaba aun fototerapia en nuestro Servicio, por lo que este procedimiento sólo pudo aplicarse en los casos de hiperbilirrubinemia de 1974 en adelante (143 casos y no en el total de 180 en que pudo realizarse exanguíneotransfusión). 
Vemos que fueron sometidos a fototerapia el $87,4 \%$ (125 casos); de los casos recibieron este tratamiento el $93,9 \%$ (31 casos) de las enfermedades hemolíticas por $\mathrm{ABO}$, el $86,4 \%$ (38 casos) de las hiperbilirrubinemias del RNPT y el $91,3 \%$ (21 casos) del grupo otros.

Fueron sometidos a exanguineotransfusión el $37,7 \%$ (68 casos); de los casos recibieron este tratamiento el $65,8 \%$ ( 27 casos) de las enfermedades hemolíticas por $\mathbf{R h}$, el $25,0 \%$ de las enfermedades hemolíticas por $\mathrm{ABO}$, el $30,6 \%$ (19 casos) de las hiperbilirrubinemias del pretérmino y el $34,4 \%$ (10 casos del grupo otros).

Es notorio que el porcentaje global de aplicación de fototerapia $(87,4 \%)$ no difiere sustancialmente de sus porcentajes de utilización en las distintas etiologías de hiperbilirrubinemia (81,4 a 93,9\%); en cambio, el porcentaje global de realización de recambios sanguíneos $(37,7 \%)$ muestra rangos muy variables $(25,0$ a $65,8 \%)$ según la etiología de la hiperbilirrubinemia, siendo claro que es la enfermedad hemolítica por $\mathrm{Rh}$ la etiología que más frecuentemente debe recambiarse, necesitando incluso 2 y 3 exanguíneotransfusiones por cada caso individual, condición que fue excepcional en las otras etiologías. Así, de 16 casos que precisaron más de un recambio, 14 correspondieron a enfermedad hemolítica por $\mathrm{Rh}, 1$ a enfermedad hemolítica por $\mathrm{ABO}$ y 1 a hiperbilirrubinemia del pretérmino.

La Tabla 3 nos muestra la distribución de las exanguíneotransfusiones a través de los 5 años revisados; es evidente, la disminución del porcentaje de casos que necesitaron ser recambiados desde 1973 (prefototerapia en nuestro Servicio) a 1977.
Tabla 3

NECESIDAD DE RECAMBIO SANGUINEO EN HIPERBILIRRUBINEMIA

\begin{tabular}{|c|c|c|c|}
\hline \multirow[t]{2}{*}{$A \bar{n} o$} & \multirow[t]{2}{*}{$\begin{array}{l}\text { Hiperbilir. } \\
N^{\circ} \text { cassis }\end{array}$} & \multicolumn{2}{|r|}{$\begin{array}{l}\text { Recambio } \\
\text { Sanguineo }\end{array}$} \\
\hline & & & $N^{\infty} \quad \%$ \\
\hline 1973 & 37 & 26 & 70,2 \\
\hline 1974 & 33 & 16 & 48,4 \\
\hline 1975 & 34 & 11 & 32,3 \\
\hline 1976 & 30 & 8 & 26,6 \\
\hline 1977 & 46 & 7 & 15,2 \\
\hline Total & 180 & 68 & 37,7 \\
\hline
\end{tabular}

La exanguineotransfusión permitió un descenso de las cifras de bilirrubinemia a un $40-60 \%$ de los valores prerrecambio, siendo lo habitual ( $90 \%$ de los casos) de un $50 \%$; la duración del procedimiento fluctuó entre 1 y $11 / 2$ hora en el $90 \%$ de los casos, presentándose cásos extremos de 35 minutos a 2 horas 25 minutos de duración en relación a $\mathrm{RN}$ de muy bajo peso o de peso muy elevado y/o dificultades técnicas durante la realización del método terapéutico.

También se consignó las complicaciones del recambio sanguíneo:

- Durante el procedimiento: sólo se presentaron dificultades técnicas, poco frecuentes, con el material ęmpleado sin repercusión sobre el paciente.

- Después del procedimiento: onfalorragia leve en 2 casos $(2,3 \%)$ que cedió con vendaje compresivo.

- Mortalidad: nula en 88 recambios efectuados (54 recambios únicos; 12 dobles y 4 triples).

En cuanto a fototerapia, la Tabla 4 muestra su rendimiento en las distintas etiologias

Tabla 4

RENDIMIENTO DE FOTOTERAPIA SEGUN ETIOLOGIA DE HIPERBILIRRUBINEMIA

\begin{tabular}{|c|c|c|c|c|c|c|}
\hline \multirow[t]{2}{*}{ Etiología } & \multicolumn{2}{|c|}{ Exito } & \multicolumn{2}{|c|}{ Fracaso } & \multicolumn{2}{|c|}{ Total } \\
\hline & $\mathrm{N}^{\circ}$ & $\%$ & $N^{\circ}$ & $\%$ & $x^{10}$ & $\%$ \\
\hline E. Hemol $x$ Rh & 16 & 51,6 & 15 & 48,4 & 31 & 100 \\
\hline E. Hemol x ABO & 29 & 82,8 & 6 & 17,2 & 35 & 100 \\
\hline Prematuridad & 29 & 76,3 & 9 & 23,7 & 38 & 100 \\
\hline Otros & 17 & 80,9 & 4 & 19,1 & 21 & 100 \\
\hline Total & 91 & 71,2 & 34 & 28,8 & 125 & 100 \\
\hline
\end{tabular}


de hiperbilirrubinemia; se consideró como éxito el que disminuyera el ritmo de ascenso de la bilirrubinemia y que no se precisara de recambio sanguíneo, y fracaso el que pese a su uso se alcanzara cifras indicatorias de exanguin eotransfusión.

Vemos que la fototerapia muestra mejor rendimiento en enfermedad hemolítica por $\mathrm{ABO}$, otros $\mathrm{e}$ hiperbilirrubinemia del pretérmino, y menor rendimiento en enfermedad hemolítica por $\mathrm{Rh} . \mathrm{El}$ rendimiento global del método es $71,2 \%$.

Para valorar, de otro modo, el rendimiento de la fototerapia por etiología de hiperbilirrubinemia, vemos en la Tabla 5 el porcentaje de exanguineotransfusiones realizadas por etiología antes (1973) y después (1974-1977) de emplearla en nuestro Servicio.

\section{Tabla 5}

PORCENTAJE DE RECAMBIOS/ETIOLOGIA ANTES Y DESPUES DE UTILIZAR FOTOTERAPIA

\begin{tabular}{|c|c|c|}
\hline \multirow[t]{3}{*}{ Etiologia } & \multicolumn{2}{|c|}{ Porcentaje de recambios } \\
\hline & Antes & Después \\
\hline & Foloterapia & \\
\hline E. Hemol. $x$ Rh & 100 & 48,4 \\
\hline E. Hemol. x ABO & 80 & 17,2 \\
\hline Prematuridad & 55,5 & 23,7 \\
\hline Otros & 66,6 & 23,8 \\
\hline
\end{tabular}

También investigamos las complicaciones de la luminoterapia; basándonos en lo que consigna la literatura al respecto, consideramos: presencia de deposiciones diarreicas de color café o verde oscuro, erupciones cutáneas diseminadas, conjuntivitis, cambio en la actividad del niño.

De los 125 casos sometidos a fototerapia en 3 de ellos $(2,4 \%)$ se presentó exantema y en 1 $(0,8 \%)$ deposiciones alteradas; estas complicaciones cedieron rápidamente al suspender el tratamiento.

El último punto que tabulamos, y el más importante ya que la finalidad del tratamiento es evitarlo, fue la presencia de daño neurológico en nuestra casuística.

De los 180 casos revisados, 6 casos $(3,3 \%)$ presentaron daño neurológico: 1 caso de enfermedad hemolítica por $\mathrm{Rh}, 2$ casos de en- fermedad hemolítica por ABO, 1 caso de hiperbilirrubinemia del pretérmino y 2 casos del grupo otros.

De ellos, 3 casos ingresaron en esa condición desde Pedro de Valdivia (2. casos) y Taltal (1 caso), y los otros 3 presentaban patología asociada grave (hipoxia perinatal + distress respiratorio en 2 casos y sepsis con compromiso meníngeo en 1 caso) lo que hace dificil valorar si el daño neurológico fue secundario a hiperbilirrubinemia o a la hipoxia e infección concomitantes.

\section{COMENTARIO}

La hiperbilirrubinemia neonatal es una patología importante durante esta etapa de la vida; tanto su magnitud como su gravedad (mortalidad, secuelas neurológicas) pueden ser disminuidas sustancialmente gracias a medidas preventivas y terapéuticas adecuadas. Entre ellas recomendamos: 1 . Control de embarazo adecuado a fin de prevenir etiologías potenciales de hiperbilirrubinemia (enfermedad hemolítica por $\mathbf{R h}$, prematuridad). 2. Atención profesional del parto para evitar partos traumáticos que, entre otras consecuencias, pueden ser causantes de hiperbilirrubinemia. 3. Diagnóstico de los casos de hiperbilirrubinemia y derivación oportuna de ellos a centros que cuenten con recursos técnicos y materiales para su tratamiento. 4. Empleo racional de los métodos terapéuticos comprobadamente eficaces en la actualidad. 5. Revisión constante de la literatura sobre el tema a fin de introducir nuevas técnicas de laboratorio o nuevos métodos terapéuticos eficaces e inocuos.

\section{RESUMEN}

Se revisaron 180 fichas clínicas de casos de hiperbilirrubinemia correspondientes a pacientes hospitalizados en la Unidad de Recién nacido Patológico y Prematuro del Hospital Regional Dr. Leonardo Guzmán de Antofagasta, durante el quinquenio 1973-1977. Se definió el criterio de selección de hiperbilirrubinemia $=$ bilirrubinemia superior a $12 \mathrm{mg} \%$ durante la primera semana de vida; se analizó las bases fisiopatológicas de los métodos terapéuticos empleados, aclarando sus normas de aplicación en el material estudiado.

Se concluyó que:

1. El $6,7 \%$ de los pacientes ingresados a la Unidad de R.N. Patológico y Prematuro corresponde a casos de hiperbili- 
rrubinema, lo que representa un $0,93 \%$ de los R.N. vivos de la ciudad de Antofagasta.

2. El $22,8 \%$ de los casos corresponde a enfermedad hemolitica por $\mathrm{Rh}$; esta etiología muestra un ascenso de su frecuencia de 1973 a 1975 , para disminuir posteriormente.

E] $26,7 \%$ de los casos corresponde a enfermedad hemolitica por $\mathrm{ABO}$; esta etiología presenta un ascenso creciente de 1973 a 1977 .

El $34,4 \%$ de los casos corresponde a hiperbilirrubinemia del pretérmino; esta etiología presenta su máximo en los primeros 2 años revisados para estabilizarse posteriormente en $27.28 \%$.

El $16,1 \%$ de los casos corresponde al rubro otros (que resume predominantemente casos de reabsorción de colecciones hemáticas); esta etiología presenta un pick en 1975, mostrando en los otros años revisados una frecuencia más o menos estable entre $12-16 \%$.

3. Las enfermedades hemolíticas por Rh y por ABO aumentan su frecuencia a medida que aumenta la edad gestacional; la hiperbilirrubinemia del pretérmino, se presentó en nuestro material, con igual frecuencia en los menores de 36 semanas y en los de 36-38 semanas de edad gestacional; el rubro otros fue más frecuente en los mayores de 38 semanas de edad gestacional.

4. La enfermedad hemolítica por Rh presentó ictericia precoz (aparecida antes de las 24 horas de vida) en $71-86 \%$ de los casos: la enfermedad hemolítica por ABO sólo en 20$60 \%$ de los casos y no hubo casos de ictericia precoz en hiperbilirrubinemia del pretérmino ni otros.

5. El porcentaje de casos de hiperbilirrubinemia sometidos a exanguineotransfusión fue disminuyendo progresivamente desde 1973 (pre-fototerapia en nuestro Servicio) a 1977 de 70,2 a $15,2 \%$.

0. Los casos de enfermedad hemolitica por Rh fueron los que se recambiaron con mayor frecuencia, necesitando incluso 2 y 3 exanguineotransfusiones por cada caso individuai, condición que fue excepcional en las otras etiologias.

7. La exanguineotransfusión permitió un descenso de las cifras de bilirrubinemia a un $40-60 \%$ de los valores prerecambio

8. La exanguineotransfusión presentó mínimas complicaciones durante $y$ después del procedimiento, y su mortalidad fue nula en 88 procedimientos realizados.

9. La fototerapia presentó mejor rendimiento en los casos de hiperbilirrubinemia por prematuridad, incompatibilidad $\mathrm{ABO}$ y otros; su rendimiento fue menor en enfermedad hemolítica por $\mathrm{Rh}$. Ef rendimiento global del método fue $71,2 \%$.

10. Las complicaciones de la fototerapia fueron muy escasas y cedieron rápidamente al suspender el tratamiento.

11. De los 180 casos revisados, 6 casos $(3,3 \%)$ presentaron daño neurológico. 3 de ellos ingresaron en esa condición desde Pedro de Valdivia (2 casos) y Taltal (1 caso), y los otros 3 presentaban patología asociada grave (hipoxia perinatal + distress respiratorio en 2 casos y sepsis con compromiso meníngeo en 1 caso) lo que hace dificil valorar si el daño neurológico fue secundario a hiperbilirrubinemia o a la hipoxia e infección concomitantes.

12. De los 180 casos revisados, $1(0,55 \%)$ falleció; correspondía a un RNPT con SDRI y enfermedad hemolítica grave sometido a exanguineotransfusión al segundo día de vida

\section{SUMMARY'}

Considering the frequency and importance of the hyperbilirubinemia during the neonatal period it was possible to determine the magnitud of this pathology in our environment, its etiology, type of patients who suffer this disease and their bilirubin curve, appraising the utility and risks of the methods used in their treatment: phototherapy and exsanguination transfusion.

For this purpose, 180 clinical filing cards of hyperbilirubinemia cases corresponding to hospitalized patients in the Pathologic and Premature Newborn Unity at Dr. Leonardo Guzmán Regional Hospital of Antofagasta during the quinquennium 1973-1977 were checked

The selection criterion of hyperbilirubin was defined as bilirubin superior to $12 \mathrm{mg} \%$ during the first week of life; the physiopathological bases of the used therapeutic mothods were analized, clarifying their application rules in the studied material.

\section{CONCLUSIONS}

1. $6.7 \%$ of the patients who entered in the Pathologic and Premature Newborn Lnity corresponds to hyperbilirubinemia cases which represents $0.93 \%$ Newborn olive in Antofagasta city.

2. $22.8 \%$ of cases with hemolytic disease by $\mathbf{R h}$; this etiology shows an ascending frequency from 1973 to 1975 being descendant since then.

$26.7 \%$ of the cases corresponds to hemolytic disease by ABO; this etiology presents a growing ascendant from 1973 to 1977 .

$34,4 \%$ of the cases corresponds to hyperbilirubinemia of the premature child, this etiology presents its highest peak in the first two years checked to become stabilized later on in $27 \%-28 \%$.

$16.1 \%$ of the cases corresponds to the title "others" (which mainly summarizes cases of reabsorption of hematic collections); this etiology presents a peak in 1975 , showing in the other revised year, a rather stable frequency between $12 \%-16 \%$.

3. The nemolytic diseases by $\mathrm{Rh}$ and $\mathrm{ABO}$ increase their frequency as the gestation age increases; the hyperbilirubinemia of the premature was presented in this material with equal frequency in the prematures who were younger than 36 weeds and in the premature of $36-38$ weeds of gestation age, the title "others" was more frequent in those older than 38 weeks of gestation age.

4. The hemolytic disease by $\mathrm{Rh}$ presented precoccious icterus (which was evident before 24 hours) in $71 \%-86 \%$ of the cases; the hemolytic disease by ABO only in $20 \%$. $60 \%$ of the cases and there was neither precoccious icterus in the premature of hyperbilirubinemia nor in others

5. The percentage of cases of hyperbilirubinemia subjected to exsanguination transfusion was progressively diminishing from 1973 (pre-phototherapy in our system) to 1977 from $70.2 \%$ to $15.2 \%$.

6. The hemolytic disease cases by $\mathrm{Rh}$ were fraquentey rechanged, two and even three exsanguination transfusions by each individual case were needed, an exceptional condition in other etiologies.

7. The exsanguination transfusion permitted to reduce 
the bilirubinemia ciphers to $40 \%-60 \%$ of the pre-rechanged) values.

8. The exsanguination transfusion presented minimal complications during and after the process and the mortality was null.

9. The phototherapy presented better yield in the hyperbilirubinemia cases by prematures, $\mathrm{ABO}$ uncongeniality and others; its yield was less in the hemolytic disease by $\mathrm{Rh}$ except in the last checked year.

10. The phototherapy complications were very scarce and they quickly diseppeared when the treatment was suspended.

11. There were 180 checked cases, 6 of them $(3.3 \%)$ presented neurologic damage, 3 of which entered in that condition from Pedro de Valdivia and Taltal (2 cases and 1 case respectively), and the other 3 presented serious associated pathology (perinatal hipoxia and respiratory distress in 2 cases and sepsis with meninx predicament in one case) what makes it hard to appreciate if the neurologic damage was secondary to hyperbilirubinemia or to the hipoxia and concomitance infection.

12. One ( $0.55 \%)$ of the 180 revised cases died, it corresponded to a RNPT with SDRI and the serious hemolytic disease submitted to exsanguination transfusion on the second day of life.

\section{REFERENCIAS}

1 Ackerman, B.D.; Dyer, G.Y.; Leydarf, M.M. Kernicterus in small premature infants. Pediatrics 45:906, vi-1970.

2 Callaham, E.W.; Thaler, M.; Karon, M.; Bauer, R.; Schmid, $R$. Phototherapy of hyperbilirubinemic infants. Pediatrics 46:481, xII, 1970.

3 Callaham, E.W.; Thaler, M.; Karon, M.; Bauer, R.; Schmid, $R$. Phototherapy of severe unconjugated hyperbilirubinemia: formation and removal of leveled bilirubin derivates. Pediatrics $46: 841$, XII, 1970.

4 Dodsan, W.E. Encefalopatía, hipoglicemia, hipocalcemia, hipomagnesemia e hiperbilirrubinemia metabólicas neonatales. Clinic. de Perinat. de N.A. p. 133, 111, 1977.

s Ertel, Iuta E. and Nezwton, William A. Therapy in congenital hyperbilirubinemia: phenobarbital and diethylnicotinamide. Pediatrics 44:43, vil, 1969.

- Fetreiro, M.; Stagno, S.; González, C. Fenobarbital asociado a fototerapia en la prevención de la hiperbilirrubinemia del niño de bajo peso. Pediatría 13:185, X-XI, 1970.

1 Ferretro, M.; Padilla, L.; Alvarez, F. Prevención de la hiperbilirrubinemia del RNPT. Influencia de la iluminación ambiental. Pediatría 16:87, vu-XII, 1973.

B Giunta, Frank and Rath, Jogeswar. Effect of enviromental illumination in prevention of hyperbilirubinemia of prematurity. Pediatrics 44:162, vIII, 1969.

9 Gartner, L.M.; Snyder, R.N.; Chabon, R.S.; Bernstein, J. Kernicterus: high incidence in premature infants. Pediatrics 45:906, vi, 1970.

10 Howard, J.; Huidobro, J.G.; Allende, N. y Sánchez, $M$. Uso de sangre heparinizada y calcificada a partir de sangre citratada (ACD) en exanguineotransfusión. Rev. Ch. de Ped., xxxvi:642, x, 1965.
1 Hodgman, J.E. and Schwartz, A. Phototherapy, and Hyperbilirubinemia of the premature. A.J. of Dis. of C'hild. 119:473, vi, 1970.

12 Lucey, J.F. Ictericia neonatal y fototerapia. Clin. Ped. de N.A. p. 827, XI, 1972.

13 Lucy, J.F. Nursery illumination and hyperbilirubinemia. Pediatrics 44:155, vin, 1969.

14 Lee, K.S.; Gartner, L.M.; Eidelmen, A.I.; Ezhuthachan $S$. Hiperbilirubinemia no conjugada en criaturas de peso muy bajo al nacer. Clin. Perinat. de N.A. p. 303, ix, 1977.

15 Lee, K.S.; Gartner, L.M. and Zarefur, I. Fluorescent dye method for determination of the bilirubin-binding capacity of serum albumin. The J. of Ped.; $86: 280$, I, 1975

16 Kaufman, N.; Kapitulnik, J. and Blondheims, S. The absorption of bilirubin by sephadex and its relationship to the criteria for exchange transfusion. Pediatrics 44: 543. x, 1969

17 Maisels, M.J. Bilirrubina: comprensión y modificación de su metabolismo en el neonato. Clin. Ped. de N.A. p. $447, \mathrm{v}, 1972$

18 Moller, J.; Ebbesen, $F$. Phototherapy in newbom in. fants with severe rhesus hemolytic disease. The $\mathrm{J}$. of Ped. 86: 135, I, 1975.

19 Obes Polleri, J. y Hill, J.S. La fototerapia en las ictericias del R.N. Rev. Ch. Ped. xxxv:638, vil, 1964.

${ }^{20}$ Sanhueza, M.Y.; Feoli, R.; Ferreiro, M. Fototerapia en la hiperbilirrubinemia del R.N.P.T. Pediatría 15: 370, VII-VIII, 1977.

21 Seligmen, J.M. Conceptos actuales cambiantes 30 bre hiperbilirrubinemia y su tratamiento en el RN. Clin. Ped. de N.A. p. 509, vI, 1977.

22 Seprílveda, M.; Mateluna, E. y Kunstman, G. Experiencias sobre exanguineotransfusión y albúmina. Rev. Ch. de Ped. xxxvi: 351, vi, 1965.

${ }^{23}$ Stem, L.; Khana, N.N.; Pharm, G.L. and Jaffe, S.J. Effect of phenobarbital on hyperbilirubinemia and glucoronide formation in newborns. A.J. of Dis. of Child. 120:26, VII, 1970.

24 Scheidt, C. Peter, David Mellits, E. and others. Toxicity to bilirubin in neonates: infant development during first year in relation to maximun neonatal serum bilirubin concentration. The J. of Ped. 91:292, vilı, 1977.

25 Teberg, A.; Hodgman, $J$. and Wu, Paul Y.K. Effoct of phototerapy on growth of low-birth-weight infants two years follow-up. The J. of Ped. 91:92, viI, 1977.

26 Tan, K.L. and others. The nature of dose response relationship of phototherapy for neonatal hyperbilirubinemia. The J of Ped. 90:448, 11I, 1977.

${ }^{27}$ Vogl, T.P. Phototherapy of neonatal hyperbilirubinemia: Bilirubin in unexposed areas of the skin. The J of Ped. 85:707, xI, 1974.

2s Vaisman, S. Hiperbilirrubinemia neonatal. Pediatría 19:224, VII-XII, 1976.

20 Wu, P.Y.K.; Lim, R.C.; Hodgman, J.E.; Kokosky, M. J. and Teberg, A.J. Effect of phototherapy in preterm infants on growth in the neonatal period. The $J$. of Ped. $85: 563, \mathbf{x}, 1974$.

30 Wallin, A.; Jalling, B. and Barens, L. Plasma concentration of phenobarbital in the neonate during prophylaxis for neonatal hyperbilirubinemia. The $\mathrm{J}$. of Ped $85: 392, \mathrm{IX}, 1974$ 